\title{
An Analytical Wall Function for 2-D Shock Wave/Turbulent Boundary Layer Interactions
}

\author{
X Wang ${ }^{1,2}$, T.J. Craft ${ }^{1}$, H. Iacovides ${ }^{1}$ \\ ${ }^{1}$ School of Mechanical, Aerospace and Civil Engineering, the University of Manchester \\ Room C9, George Begg Building, Manchester, UK \\ ${ }^{2}$ China Aerodynamic Res \& Dev Ctr \\ Mianyang, 621000, Sichuan, People R China \\ xinguang.wang@manchester.ac.uk; tim.craft@ manchester.ac.uk; h.iacovides@ manchester.ac.uk
}

\begin{abstract}
When handling the near-wall regions of turbulent flows, it is necessary to account for the viscous effects which are important over the thin near-wall layers. Low-Reynolds-Number turbulence models do this by including explicit viscous and also damping terms which become active in the near-wall regions, and using very fine near-wall grids to properly resolve the steep gradients present. In order to overcome the cost associated with the low-Re turbulence models, a more advanced wall function approach has been implemented within OpenFoam, and tested together with a standard log-law based wall function in the prediction of flows which involve 2-D shock wave/turbulent boundary layer interactions (SWTBLIs). On the whole, from the calculation of the impinging shock interaction, the three turbulence modelling strategies, the Lauder-Sharma $k-\varepsilon$ model with Yap correction (LS), the high-Re $k-\varepsilon$ model with standard wall function (SWF) and analytical wall function (AWF), display good predictions of wall-pressure. However, the SWF approach tends to underestimate the tendency of the flow to separate as a result of the SWTBLI. The analytical wall function on the other hand, is able reproduce the shock-induced flow separation and returns predictions similar to those of the low-Re model, using a much coarser mesh.
\end{abstract}

Keywords: SWTBLIs; Skin-friction, Turbulence modeling, Wall function

\section{Introduction}

One of the common occurrences in supersonic flow is shock wave/turbulent boundary layer interactions (SWTBLIs), which include significant change of temperature, density, velocity and Mach number near the wall. In CFD, turbulence modelling is a key element in the aerodynamic design of advanced aircraft, especially in the complex flow simulations such as those in the wing-body junction and inlet of airbreathing propulsion systems, where SWTBLIs happen. There are hundreds of experiments of SWTBLIs, some of which have been summarized by Settles and Dodson [1] .

For the weaker interactions (see Fig. 1 left), the boundary layer remains attached, and the interaction is embedded well in the boundary layer. Green [2] studied cases at Mach 2.5, $\operatorname{Re}_{\theta}$ of $4 \times 10^{5}$, deflection angles of $2^{\circ}, 2.5^{\circ}$ and $5^{\circ}$ and showed that the reflected wave is a single shock with equal and opposite reflection angle to that of the incident wave. For stronger interactions (see Fig. 1 right), the oblique shock is strong enough to cause separation of the turbulent boundary layer. The increase of pressure which takes place at separation, generates the leading reflected shock $\mathrm{C} 2$. Then shock $\mathrm{C} 2$ intersects the oblique shock $\mathrm{C} 1$ at point $\mathrm{H}$, from which emanate the two refracted shocks $\mathrm{C} 3$ and $\mathrm{C} 4$. The point $\mathrm{H}$ could be seen clearly in the Schlieren pictures ${ }^{[2]}$ at deflection angles of $6.5^{\circ}, 8^{\circ}, 9.5^{\circ}$ and $10.5^{\circ}$.

For the accuracy of numerical results, the treatment of the sublayer, which is very close to the solid wall, plays an important role, since molecular diffusion results in the significant transport of heat and momentum. There are two broad strategies to the near wall turbulence modelling, one of which is based on the use of Low-Reynolds-Number models which requires the use of fine near-wall meshes to resolve the significant changes within the sublayer. As a result, most of the computation time is utilized in the resolution of the near-wall region, which may only be a small region, with the sublayer possibly covering only around $1 \%$ of the computation domain. In order to overcome this expense, and sometimes convergence difficulties associated with very high aspect ratio near-wall cells, the wall-function approach is widely used in advanced CFD codes, because of its robustness, fast convergence rate, and reasonable accuracy in a number of situations. 

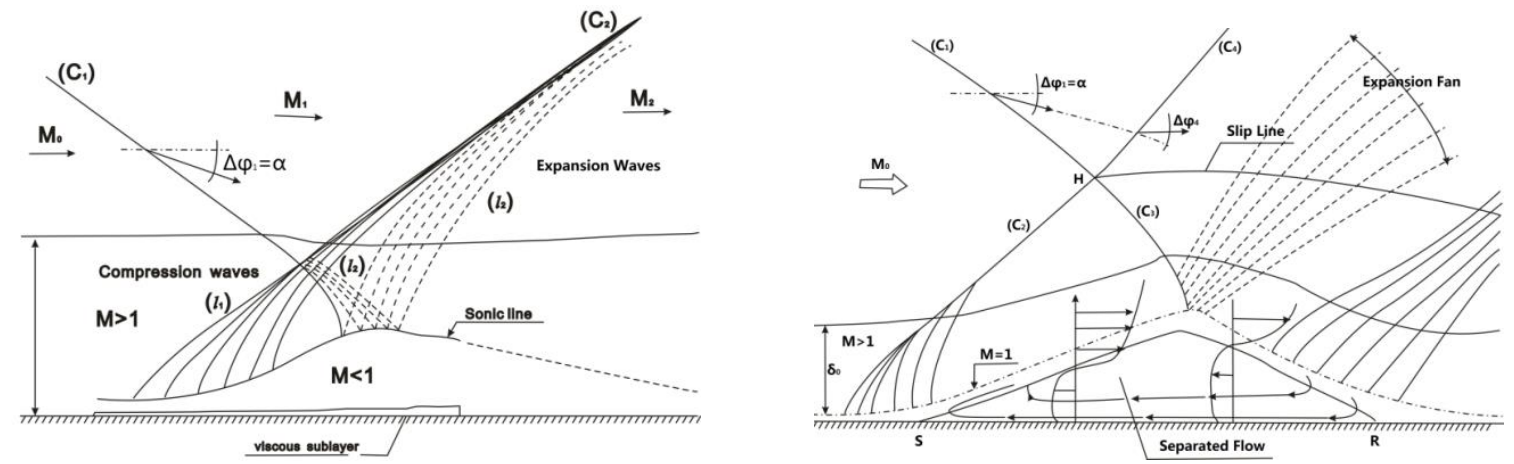

Fig. 1: Shock reflection without (left) and with (right) boundary-layer separation ${ }^{[3]}$.

There are different types of wall functions to bridge the gap between the wall and the fully turbulent area. However, most existing wall functions are based on the log-law under local-equilibrium flow conditions. In this research, the main aim is to examine the performance of wall-function treatments which have been successfully used in a range of incompressible flows, and explore how compressibility effects could be accounted for in such approaches. The objective is to utilize these advanced wall-functions to capture the rapid changes and turbulence properties in the near-wall region with fast convergence rate, robustness and reasonable accuracy. In order to implement the wall functions, the open source CFD package OpenFoam v2.3.1 which employs the finite volume method. A number of experimental and DNS cases of 2-D impinging shock are selected for validation purposes.

\section{Physical Model}

The Favre-Reynolds averaged Navier-Stokes equations are used for the governing equations. When the thermally and calorically perfect gas assumption is made, the equation of state can be taken as $p=\rho R T$. The molecular viscosity is calculated by the Sutherland equation[4].

Two RANS models are used in the calculation. One of them is the standard k- $\varepsilon$ model[5], which is a high Reynolds number model. The standard $\mathrm{k}-\varepsilon$ model described above is only valid for fully turbulent flow. For flows involving solid walls, the local Reynolds number, Rt $(=\mathrm{k} 2 / \mathrm{v} / \varepsilon)$ can be small enough that viscous effects cannot be neglected. Launder and Sharma[6] employed damping functions dependent on Rt for the resolution of near-wall turbulent length scales. In separated flows, the $\varepsilon$ equation returns high levels of near wall turbulence. In order to address this problem, the Yap correction [7] is added as an extra source term to modify the $\varepsilon$ equation.

\section{Wall Function}

\subsection{Standard Wall Function}

In local equilibrium turbulent boundary layers, the law of the wall applies. We can write this as:

$$
\begin{aligned}
U^{*} & =\frac{1}{\kappa} \log \left(E y^{*}\right) \text { when } y^{*}>y_{v}^{*} \\
U^{*} & =y^{*} \text { when } y^{*} \leq y_{v}^{*}
\end{aligned}
$$

where $U^{*}$ and $y^{*}$ are defined as

$$
U^{*}=U k_{\mathrm{v}}^{1 / 2} /\left(\tau_{\mathrm{w}} / \rho\right), y^{*}=y k_{\mathrm{v}}^{1 / 2} / v
$$

In (1), $\kappa=0.4178, E=9.793$ and $y_{v}^{*}=20$, Normally, in the near-wall sublayer, momentum transfer is dominated by molecular diffusion and heat conduction. The temperature log law can be expressed similar to that for $U^{*}$. Normally, the turbulent kinetic energy $k$ is assumed to be constant across the near-wall fully turbulent region, so $k_{v}$ is the same as $k_{P}$ (where $\mathrm{P}$ denotes the near wall nodes). For the energy equation: 


$$
T^{*}=\frac{\left(T_{\mathrm{w}}-T_{\mathrm{p}}\right) \rho c_{\mathrm{p}} C_{\mu}^{1 / 4} k_{\mathrm{p}}^{1 / 2}}{\dot{q}}
$$

where $\dot{q}$ is the heat flux, $c_{p}$ is the heat capacity at constant pressure, and $C_{\mu}=0.09$. In order to get the turbulent kinetic energy at near wall cell $\mathrm{P}$, the transport equation of $\mathrm{k}$ should be solved in the near-wall cell. Since the production and dissipation of $k$ can both vary significantly in this region, suitable approximations should be made for the cell-averaged source and sink terms, and the cell-averaged generation and dissipation rates for $k$ are then used in discretizing the $k$ equation. Using assumptions based on those above, of a simple boundary layer in local equilibrium, the cell-averaged generation and dissipation rates for $k$ over the near-wall cell can be approximated as:

$$
\begin{gathered}
\bar{P}_{k}=\frac{\tau_{\mathrm{w}}^{2}}{\kappa c_{\mu}^{1 / 4} \rho k_{\mathrm{p}}^{1 / 2} y_{\mathrm{n}}} \log \left(y_{\mathrm{n}} / y_{\mathrm{v}}\right) \\
\bar{\varepsilon}=\frac{1}{y_{\mathrm{n}}}\left[\frac{2 k_{\mathrm{p}}}{y_{\mathrm{v}} / v}+\frac{k_{\mathrm{p}}^{3 / 2}}{c_{l}} \log \left(y_{\mathrm{n}} / y_{\mathrm{v}}\right)\right]
\end{gathered}
$$

The wall shear stress from the log-law properties evaluated at the near-wall node $\mathrm{P}$ is expressed as:

$$
\tau_{\mathrm{w}}=\frac{C_{\mu}^{1 / 4} \rho \kappa U_{P} k_{P}^{1 / 2}}{\log \left(E^{*} y^{+}\right)}
$$

The cell-averaged production and dissipation rates were calculated by assuming constant shear stress and a linear turbulence length scale variation across the near-wall cell.

\subsection{Analytical Wall Function}

The AWF ${ }^{[8]}$ is based on the analytical solution of the simplified Reynolds equations, and takes convection and pressure gradients into account. In the near wall region, the transport equations are simplified by the boundary layer assumptions. Then the simplified momentum and temperature Reynolds equations for a forced convection flow in the near wall $x-y$ plane can be written as:

$$
\begin{gathered}
\frac{\partial(\rho U U)}{\partial x}+\frac{\partial(\rho V U)}{\partial y}=-\frac{d P}{d x}+\frac{\partial}{\partial y}\left[\left(\mu+\mu_{t}\right) \frac{\partial U}{\partial y}\right] \\
\frac{\partial(\rho U T)}{\partial x}+\frac{\partial(\rho V T)}{\partial y}=\frac{\partial}{\partial y}\left[\left(\frac{\mu}{\operatorname{Pr}}+\frac{\mu_{t}}{\operatorname{Pr}_{t}}\right) \frac{\partial T}{\partial y}\right]
\end{gathered}
$$

A simple prescription of turbulent viscosity can be expressed as:

$$
\begin{gathered}
\mu_{t}=0 \text { when } y<y_{v} \\
\mu_{t}=\mu \alpha\left(y^{*}-y_{v}^{*}\right)=\mu c_{\mu} c_{l}\left(y^{*}-y_{v}^{*}\right) \text { when } y_{n}>y>y_{v}
\end{gathered}
$$

where $c_{\mu}$ and $c_{l}$ are the same as described in the one-equation turbulence model, so $\alpha=c_{\mu} c_{l}$ is 0.2295 . The simplification assumptions described above make it possible to solve the momentum and energy equation analytically.

Equation (7) can be rewritten using the dimensionless wall distance, $y * a s$ : 


$$
\frac{\partial}{\partial y^{*}}\left[\left(\mu+\mu_{t}\right) \frac{\partial U}{\partial y^{*}}\right]=C
$$

where $C$ is the term which includes the effect of the pressure gradient and convection in (11). The convection part in $C$ is approximated by the analytical solution at previous time step.

Thus in the viscous sublaye $\left(y^{*}<y_{v}^{*}\right)$, the first and second integration of (11) using (9) and (10) is:

$$
\begin{gathered}
\mu \frac{\partial U_{1}}{\partial y^{*}}=C_{1} y^{*}+A_{1} \\
\mu U_{1}=\frac{C_{1}}{2} y^{* 2}+A_{1} y^{*}+B_{1}
\end{gathered}
$$

In the fully turbulent region $\left(y^{*}>y_{v}^{*}\right)$, after the integration the derivative of velocity and the velocity are given as:

$$
\begin{gathered}
\mu \frac{\partial U_{2}}{\partial y^{*}}=\frac{C_{2} y^{*}+A_{2}}{1+\alpha\left(y^{*}-y_{v}^{*}\right)} \\
\mu U_{2}=\frac{C_{2}}{\alpha}\left[y^{*}-\left(\frac{1}{\alpha}-y_{v}^{*}\right) \ln Y\right]+\frac{A_{2}}{\alpha} \ln Y+B_{2}
\end{gathered}
$$

Finally, the wall shear stress can be obtained

$$
\tau_{\text {wall }}=-\left.\mu \frac{\partial U}{\partial y}\right|_{y=0}=-\frac{\rho \sqrt{k_{P}}}{\mu} A_{1}
$$

An approximation for the cell-averaged production of turbulence kinetic energy can be obtained by

$$
\overline{P_{k}}=-\frac{1}{y_{n}} \frac{\rho \sqrt{k_{P}}}{\mu} \int_{y_{v}^{*}}^{y_{n}^{*}} \mu \alpha\left(y^{*}-y_{v}^{*}\right)\left(\frac{\partial U_{2}}{\partial y^{*}}\right)^{2} d y^{*}
$$

This term is needed for the kinetic energy transport equation to represent the production of the kinetic energy in a consistent way.

The expression of mean dissipation rate across the cell has been also modified. From equations (8) and (9), Chieng and Launder[9] had assumed a two-part dissipation profile, which could be rewritten here in the turbulent region as:

$$
\varepsilon=\frac{k^{3 / 2}}{c_{l} y}
$$

Jones and Launder ${ }^{[10]}$ had approximated the exact solution in the viscous layer as:

$$
\varepsilon=2 v\left(\frac{\partial k^{1 / 2}}{\partial y}\right)^{2} \approx \frac{2 v k}{y^{2}}=\frac{2 v k_{P}}{y_{v}^{2}}
$$


In order to avoid the discontinuity of dissipation rate of turbulence kinetic energy in Fig 2(a), a position is selected as in Fig 2(b) to make $\varepsilon$ continuous at the surface.

$$
\frac{k_{P}^{3 / 2}}{c_{l} y_{d}}=\frac{2 v k_{P}}{y_{d}^{2}} \text { or } y_{d}^{*}=2 c_{l}=5.1
$$

The mean dissipation rate can then be obtained by integrating this two-part variation to obtain:

$$
\bar{\varepsilon}=\frac{1}{y_{n}}\left[\frac{2 k_{P}^{3 / 2}}{y_{d}^{*}}+\frac{k_{P}^{3 / 2}}{2.55} \ln \left(\frac{y_{n}}{y_{d}}\right)\right]
$$

There is now only one remaining constant, namely the non-dimensional viscosity sublayer thickness $y_{v}^{*}$. From the numerical experiments for fully developed pipe flow, this value is determined as 10.1

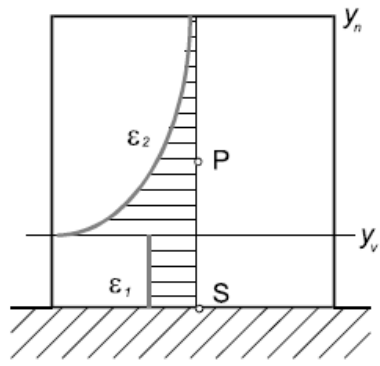

(a)

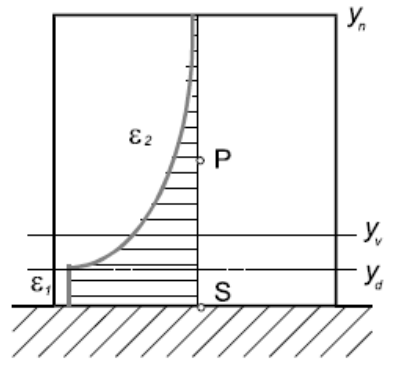

(b)

Fig. 2: Sketch map of $\varepsilon$ for (a) the standard and (b) the proposed wall function.

From (8), the wall temperature, needed to complete the thermal wall function, can be obtained from the same procedures as:

$$
T_{\text {wall }}=T_{n}-\frac{\operatorname{Pr}_{v}}{\mu_{v}}\left[\frac{C_{t h 2}}{\alpha_{t}}\left(y_{n}^{*}-y_{v}^{*}\right)+\frac{\ln Y_{T n}}{\alpha_{t}}\left(A_{t h 1}+C_{t h 1} y_{v}^{*}-\frac{C_{t h 2}}{\alpha_{t}}\right)+y_{v}^{*}\left(\frac{C_{t h 1}}{2} y_{v}^{*}+A_{t h 1}\right)\right]
$$

and the wall heat flux is related to the wall temperature by following expression:

$$
q_{\text {wall }}=-\frac{\rho c_{p} \sqrt{k_{P}}}{\mu} A_{t h 1}
$$

More detail of refinement and implementation of the AWF can be found in [7][11][12].

\section{Results}

The experimental test case ${ }^{[13]}$ of an impinging shock is used here to test some turbulence models in OpenFoam. The shock turning angles are $13^{\circ}, 10^{\circ}$, and $7^{\circ}$, to obtain different separation zones depending on the impinging shock strength. This case is referred to as the High-Reynolds-number (HR) case. Another case will be referred to as the Low-Reynoldsnumber (LR) case $^{[14]}$.

\subsection{High-Reynolds-Number (HR) Case}

Structured grids are used for this simple geometry, uniform in the streamwise direction and refined normal to the wall, and the value of $y+$ is about 15 for the high-Reynolds-number turbulence model with wall function approaches, and 0.6 for 
the low-Reynolds-number turbulence models. The computational domains and a sample grid are shown in Figure 3, where LR denotes the low-Re case and HR denotes the high-Re case.

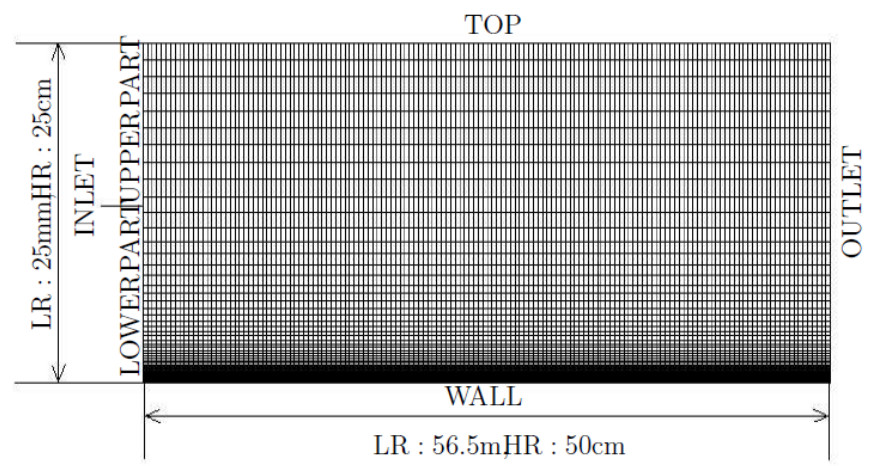

Fig. 3: Computational domains for 2-D impinging shock interaction.

The experimental inflow conditions for this case are listed in Table 1, where $\operatorname{Re}_{\theta 0}$ denotes the Reynolds number based on the momentum thickness $\theta_{0}$, and $\delta_{0}$ denotes the boundary thickness. The lower part of inlet boundary profile is a developed turbulent boundary layer from the calculation of flat plate flow at momentum thickness $\theta_{0}=0.082 \mathrm{~cm}$ with $1.5 \%$ freestream turbulence intensity and a ratio of $\mu_{t} / \mu=10$. In the upper part of the inlet boundary, the inviscid oblique shock relation equations are used to calculate the flow variables downstream of the shock. At the wall, an isothermal no-slip wall boundary condition is used. At top and outlet boundaries, Neumann conditions are used for all components.

Table 1: Inflow conditions for high-Re case.

\begin{tabular}{|c|c|c|c|c|c|}
\hline$M_{\infty}$ & $\operatorname{Re}_{\theta 0}$ & $\theta_{0}, \mathrm{~cm}$ & $\delta_{0}, \mathrm{~cm}$ & $p_{t \infty}, N / m^{2}$ & $T_{t \infty}, \mathrm{K}$ \\
\hline 2.9 & 47000 & 0.082 & 1.69 & 689010 & 291 \\
\hline
\end{tabular}

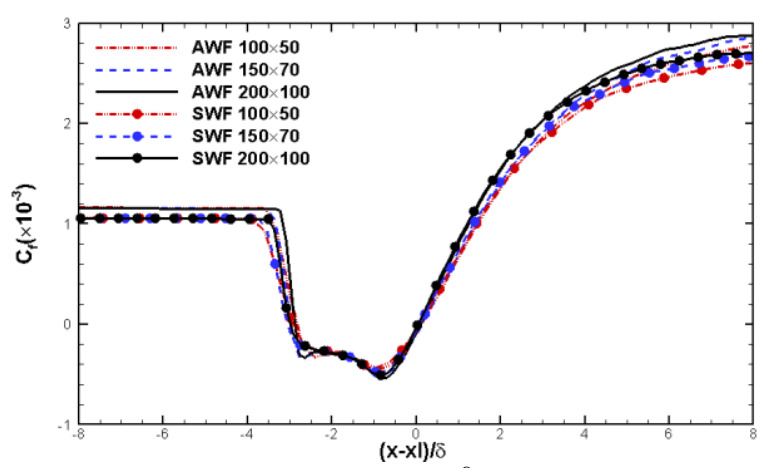

Fig. 4: Grid independency study for the high Reynolds number $13^{\circ}$ impinging shock interaction at Mach=2.9, $\operatorname{Re}_{\theta 0}=47000$.

Figure 4 shows grid-independence studies for the $k-\varepsilon$ model using analytical wall function and standard wall function, and the results indicate that the meshes used result in grid-independent solutions. Figure 5 compares the numerical results of wall pressure and skin friction with corresponding experimental variations.

For the $7^{\circ}$ case, the wall pressure predicted by all models agrees well with the experimental measurements. There is no separation from the numerical or experimental results indicated by the skin friction $C_{f}$. The results of the $k-\varepsilon$ model with standard wall function have the largest skin-friction in the interaction region, while the results of the $k-\varepsilon$ model with analytical wall function have the smallest skin-friction, which is closer to the experiment at Mach=2.9, $\operatorname{Re}_{\theta 0}=47000$.

For the $10^{\circ}$ case, all the models return similar wall pressure, which fits the experimental data well. From the skin friction results, the numerical and experimental data show that there is a small separation zone near the inviscid 
impingement point. The results of the Lauder-Shamma (LS) model are in good agreement with the experiment, the results of the $k-\varepsilon$ model with analytical wall function are close to the results of the low-Re number model (LS), and the standard wall function approach tends to underestimate the skin friction in the interaction zone.

For the $13^{\circ}$ case, generally, all the models give good prediction to the wall pressure, but slight differences are present near the beginning of the interaction. The LS model tends to predict the start point of interaction closer to the experiment than the wall function approaches. Standard wall function approach gives closer results to the LS model than the analytical wall function approach, but two different wall function approaches do not have much difference. From the skin friction, the results of all models fit the experiment well, and bigger separation bubble is predicted by the LS model, while the wall function approaches give similar size with each other. From all the comparisons, the skin friction of analytical wall function approach tends to give closer results to the LS model, and is closer to the experiment, while the standard wall function approach tends to underestimate the strength of interaction.
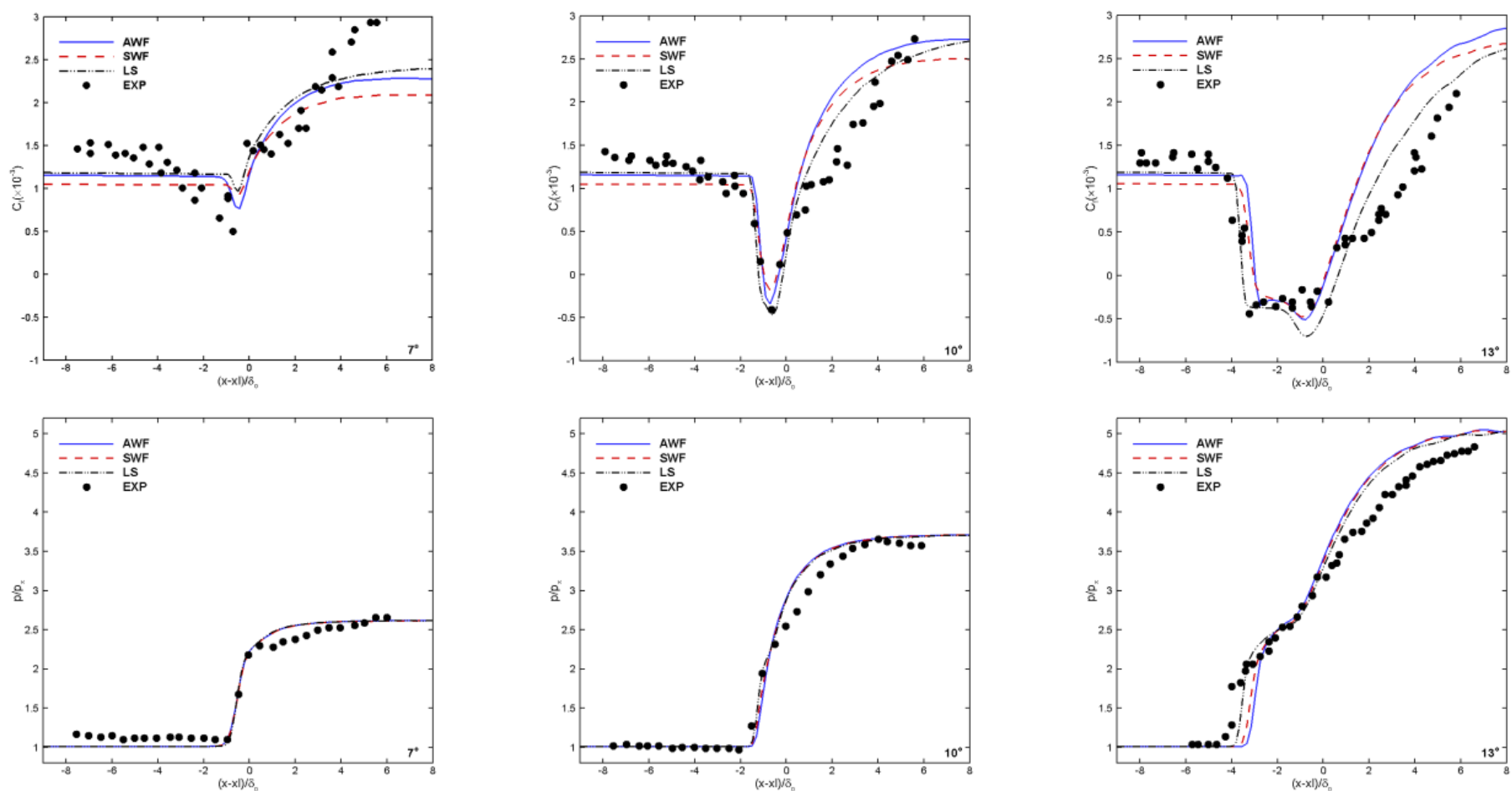

Fig. 5: Mean wall pressure (bottom) and skin-friction (top) distribution at different impinging angles $13^{\circ}\left(\right.$ left), $10^{\circ}$ (middle) and $7^{\circ}$ (right) at $\mathrm{Mach}=2.9, \operatorname{Re}_{\theta 0}=47000$.

Figure 6 displays the mean velocity comparison at eight different streamwise locations. In general, all the models give good predictions at different locations. Locations 1 and 8 are located outside of the separation zone, and similar velocity results are predicted. At location 2, the LS model predicts the flow has separated, while the results of the wall function approaches and experiment still show unseparated flow.

Figure 7 shows the comparison of Reynolds streamwise stress at the same eight different streamwise locations as in Figure 5. From the results, the numerical results underestimate the Reynolds streamwise stress at eight all locations, especially in the middle of separation zone. This underestimation have been explained by Chassaing [15], who has shown that the low-frequency oscillations, which eventually present in the experimental flow, will be considered as turbulence by the experimental averaging procedure. 

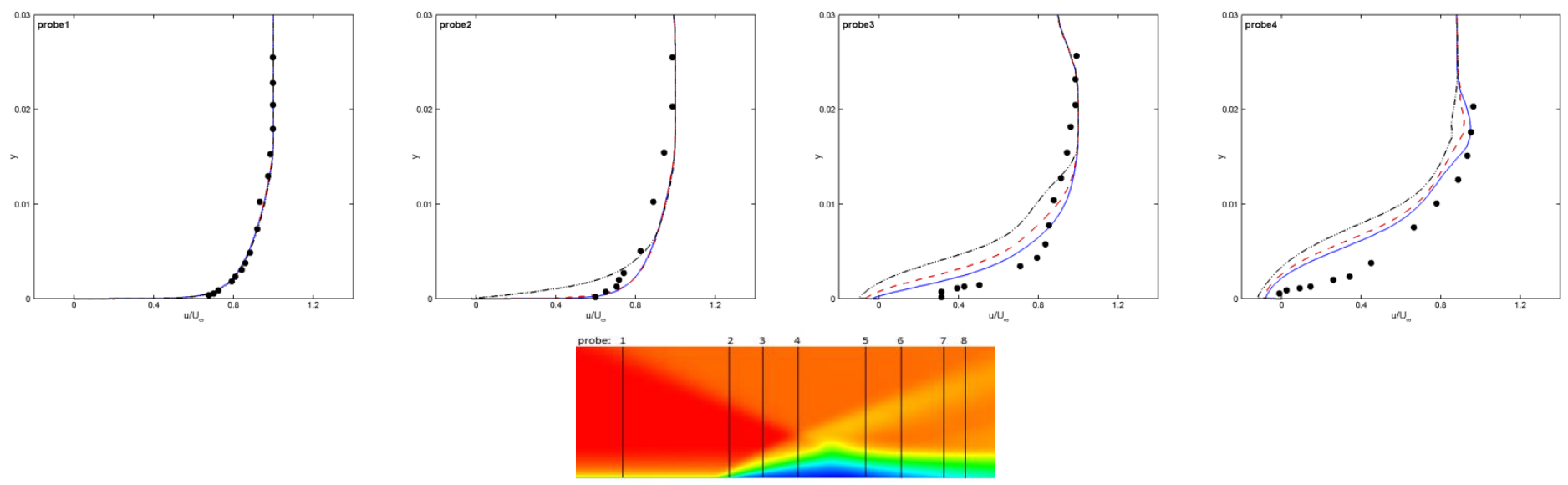

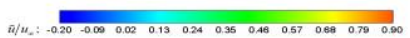
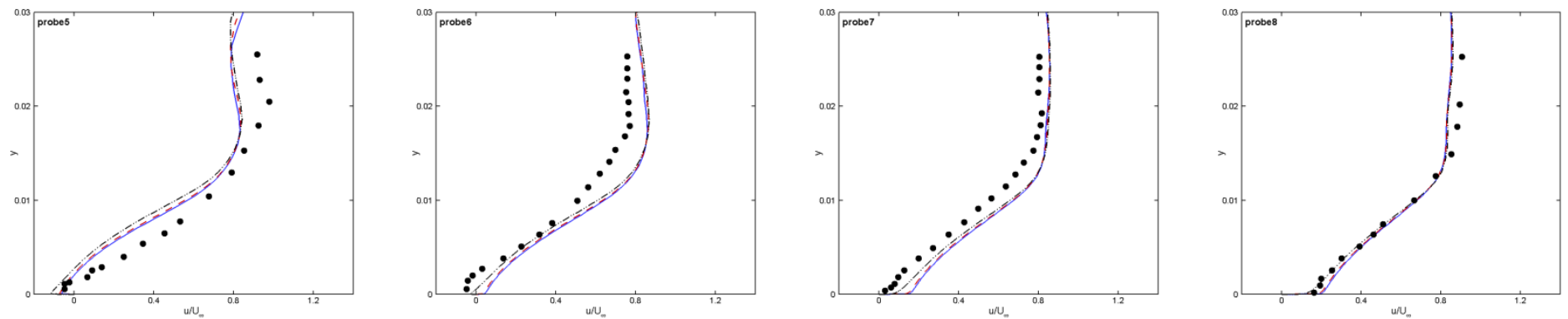

Fig. 6: Comparison of mean velocity profiles for the $13^{\circ} \mathrm{High}-$ Re impinging shock interaction at eight streamwise locations. AWF (blue solid line), SWF (red dashed line), LS model (black dashed-dot-dot line) and experiment (black circle) at Mach=2.9, $\operatorname{Re}_{\theta 0}=47000$.
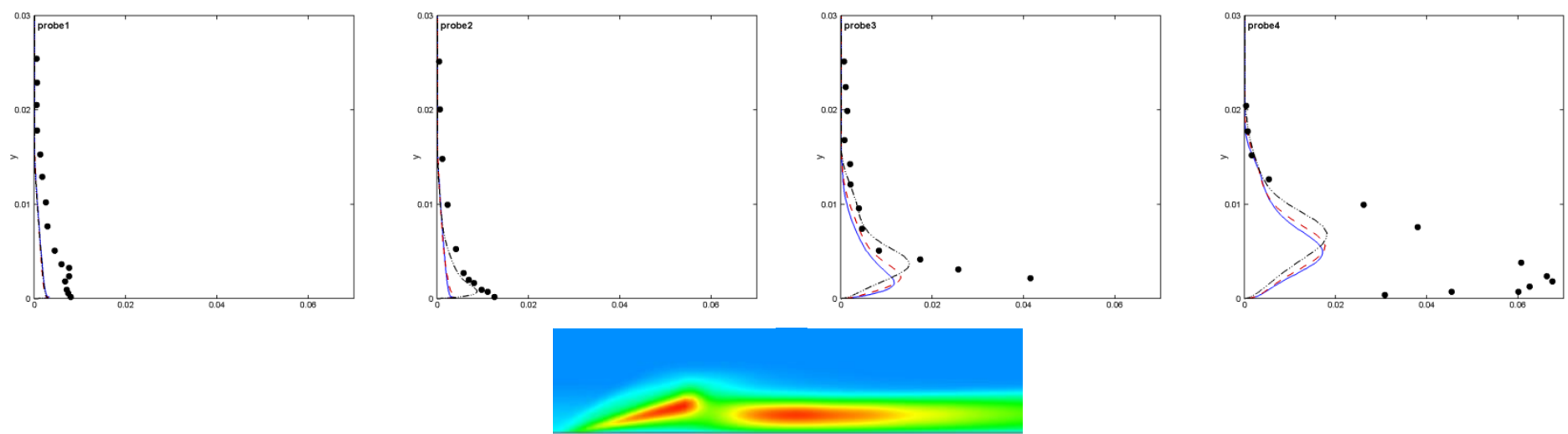

$u^{\prime \prime \prime} u^{\prime \prime} / u_{\infty}^{2}:-0.003 \quad 0.0014 \quad 0.0058 \quad 0.01020 .0146 \quad 0.019$
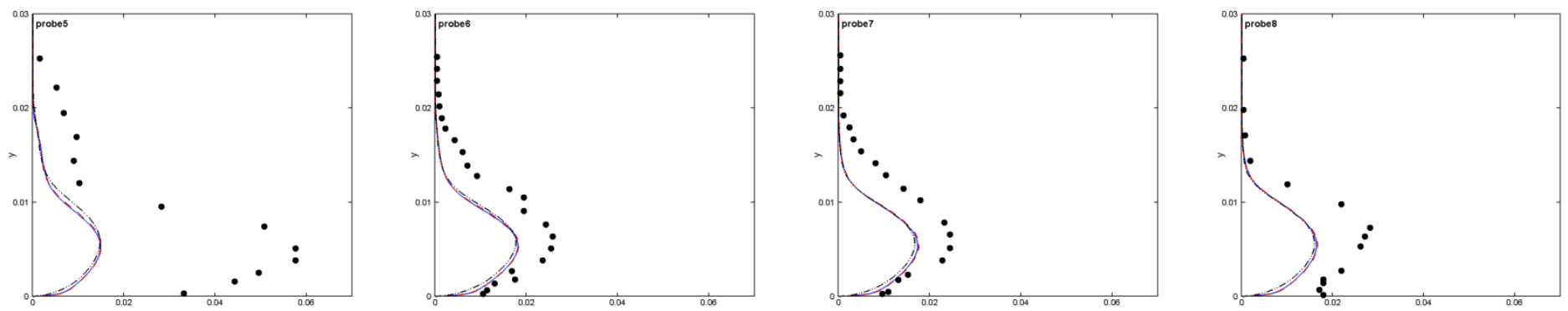

Fig. 7: Comparison of Reynolds streamwise stress for the High-Re impinging shock interaction at eight streamwise locations. AWF (blue solid line), SWF (red dashed line), LS model (block dashed-dot-dot line) and experiment (black circle) at Mach=2.9, $\operatorname{Re}_{\theta 0}=47000$. 


\subsection{Low-Reynolds-Number (HR) Case}

The DNS inflow conditions for this case are listed in Table 2. These will be used in the upper part of the inlet boundary, and the lower part of inlet boundary profile is a developed turbulent boundary layer from the calculation of flat plate flow at momentum thickness $0.147 \mathrm{~mm}$.

Table 2: Inflow conditions for low-Re case.

\begin{tabular}{|c|c|c|c|c|c|}
\hline$M_{\infty}$ & $\operatorname{Re}_{\theta 0}$ & $\theta_{0}, \mathrm{~mm}$ & $\delta_{0}, \mathrm{~cm}$ & $p_{t \infty}, N / m^{2}$ & $T_{t \infty}, \mathrm{K}$ \\
\hline 2.25 & 3725 & 0.147 & 2 & 23813 & 169.4 \\
\hline
\end{tabular}

The computational domains and a sample grid are shown in Figure 4, where structured grids are used, $\mathrm{y}+=15$ for the high-Re k- $\varepsilon$ model with wall function approaches, and $y+=0.6$ for the LS model. The boundary conditions are similar to those of the high Reynolds impinging shock interaction, except an adiabatic condition is used for the wall temperature. From the grid-independence studies in Figure 8, the results of the k- $\varepsilon$ model using analytical wall function and standard wall function converge when the grid number increases.

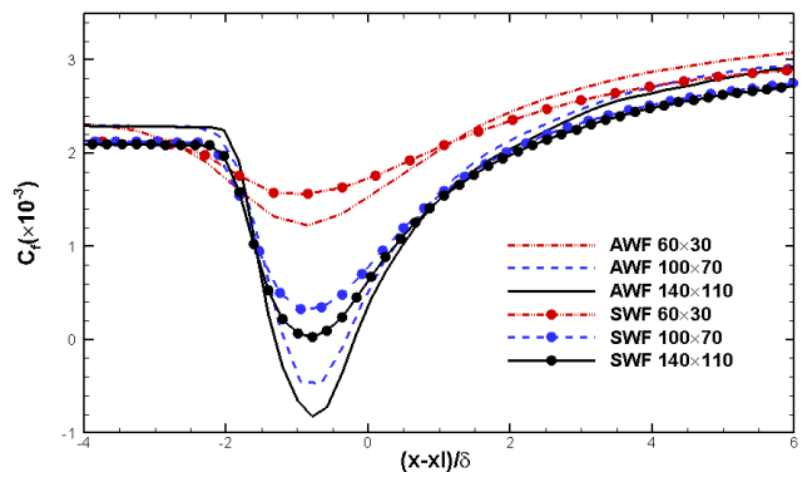

Fig. 8: Grid independency study for the low Reynolds number 130 impinging shock interaction at $M a c h=2.25, \operatorname{Re}_{\theta 0}=3725$.

Figure 9 displays the wall pressure and skin friction comparisons with the DNS results and experiment. The SWF approach fails to predict a separation zone, while the LS model returns a larger separation zone than the DNS results. The AWF approach returns smaller zone than the DNS results, since the beginning of interaction is located downstream to the results of DNS, as can be seen from the surface pressure and skin-friction. However, the reattachment point of the AWF fits well with the LS and experimental results. In this case, the SWF underestimates the SWTBLI and fails to reproduce the separation bubble in the near wall area. Downstream of the interaction zone, all the three models capture the recovery of skin-friction, and are in good agreement with the DNS results, though they are a bit further from the experimental results.
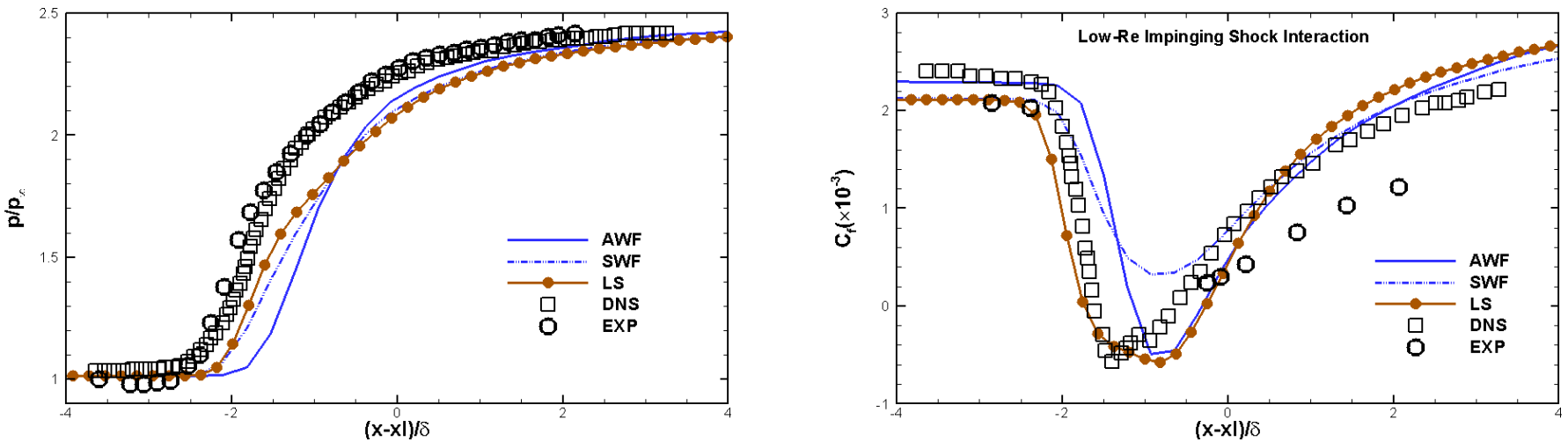

Fig. 9: Mean wall pressure(left) and Skin-friction (right) distribution comparison with DNS results and experiment for low-Re impinging shock interaction at $\mathrm{Mach}=2.25, \mathrm{Re}_{\theta 0}=3725$. 
Figure 10 shows comparisons of mean streamwise velocity profiles for the low Reynolds number impinging shock at these six locations. At location 1, which is located upstream of the interaction zone, the undisturbed turbulent boundary layers by using the RANS models are in good agreement with the DNS one. At location 4, downstream of the separation point, the LS model predicts a thicker boundary layer, while use of the AWF and SWF result in the prediction of a boundary layer thickness comparable to that of the DNS. At location 6, further downstream within the separation bubble, the AWF predicts a thinner boundary layer than the LS and SWF ones. At location 9, the velocity distribution is quite similar to location 6. At locations 10 and 13, downstream of the reattachment point, the boundary layer relaxes to its equilibrium state, and all three models are in good agreement with the DNS data.
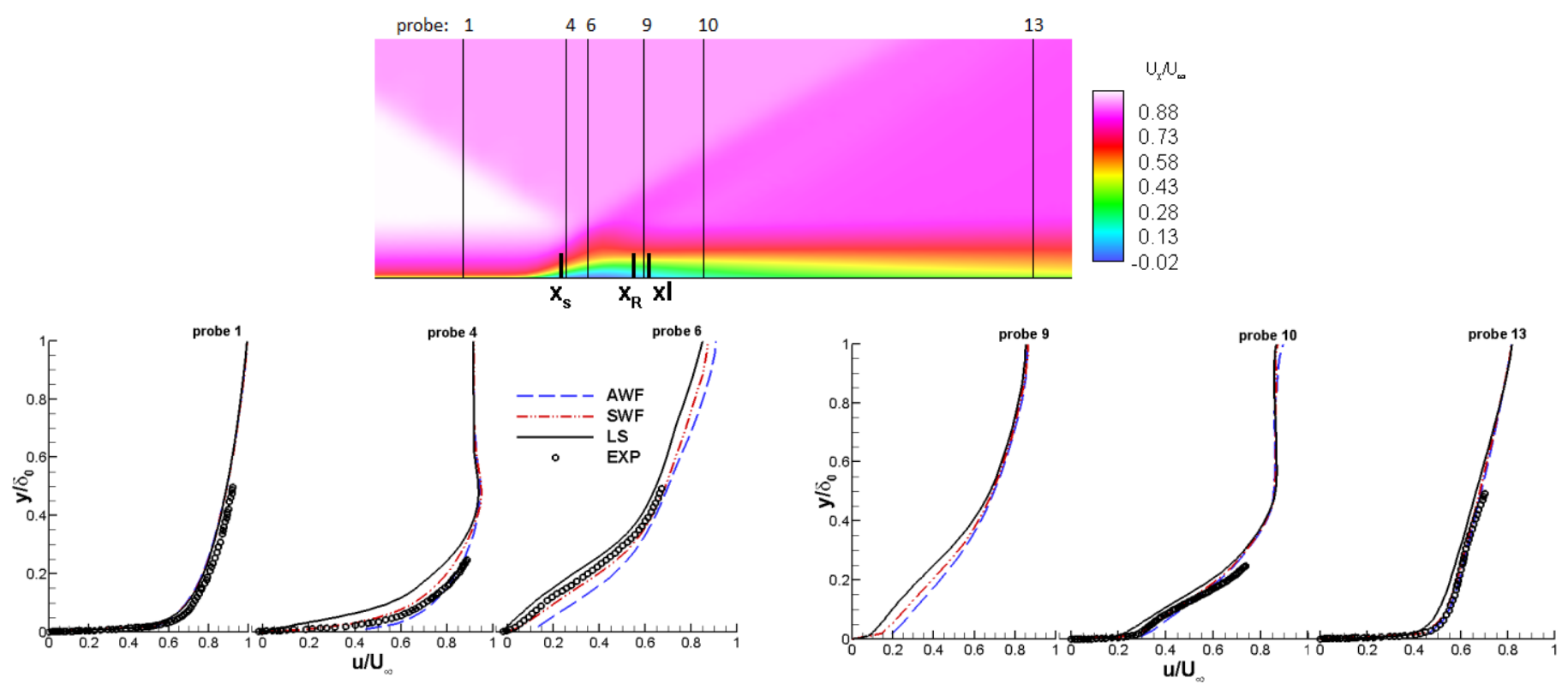

Fig. 10: Comparison of mean streamwise velocity profiles for the low Reynolds number impinging shock at six streamwise locations at $\mathrm{Mach}=2.25, \operatorname{Re}_{\theta 0}=3725$.

\section{Conclusion}

In this paper, the LS model, and the high-Re $k-\varepsilon$ model with standard and analytical wall functions have been tested by impinging shock interaction flows at two different Reynolds numbers. The main conclusions are:

(1) For the high-Re case, the wall pressure, predicted by all three models is in good agreement with the experiment. The skin friction predicted by analytical wall function approach tends to give results closer to those of the LS model, and also those of the experiment, while the standard wall function approach tends to underestimate the strength of interaction.

(2) All the models give good predictions of mean velocity at all eight locations. The numerical methods underestimate the Reynolds streamwise stress at all eight locations, especially in the middle of separation zone.

(3) For the low-Re case, the SWF underestimates the SWTBLI and fails to reproduce the separation bubble in the near wall area from the skin friction comparison. The mean streamwise velocity results by using the RANS models are in good agreement with the DNS results.

On the whole, from the calculation of the impinging shock interaction, all three models display a good prediction of wall-pressure. However, the SWF approach tends to underestimate the SWTBLI, and for the low-Re impinging shock interaction case, where there is a small separation, it fails to reproduce the separation bubble. The analytical wall function of Craft et al (2002) on the other hand, is able to reproduce the shock-induced flow separation and returns predictions similar to those of the low-Re model, without the need for fine resolution of the near-wall region. 


\section{References}

[1] G. S. Settles, L. J. Dodson, "Supersonic and hypersonic shock/boundary-layer interaction database," AIAA Journal, vol. 32, no. 7, pp. 1377-1383, 1994.

[2] J. E. Green, "Reflexion of an oblique shock wave by a turbulent boundary layer," Journal of Fluid Mechanics, vol. 40, no. 1 , pp. 81-95,1970.

[3] L. J. Souverein, "On the Scaling and Unsteadiness of Shock Induced Separation," Ph.D. Thesis, Faculty of Aerospace Engineering, Delft Univ. of Technology, Delft, the Netherlands, 2010.

[4] W. Sutherland, "The viscosity of gases and molecular force," Philosophical Magazine Series 5, vol. 36, no. 223, pp. 507-531, 1893.

[5] B. E. Launder, D. B. Spalding, "The numerical computation of turbulent flows," Computer Methods in Applied Mechanics and Engineering, vol. 3, no. 2, pp. 269-289, 1974.

[6] B. E. Launder, B. I. Sharma, "Application of the energy-dissipation model of turbulence to the calculation of flow near a spinning disc," Letters Heat Mass Transfer, vol. 1, pp. 131-137, 1974.

[7] C. R. Yap, "Turbulent heat and momentum transfer in recirculating and impinging flows," Ph.D. thesis, University of Manchester Institute of Science and Technology (UMIST), 1987.

[8] T. J. Craft, A. V.Gerasimov, H. Iacovides, B. E. Launder, "Progress in the generalization of wall-function treatments," International Journal of Heat and Fluid Flow, vol. 23, no. 2, pp. 148-160, 2002.

[9] C. C. Chieng, B. E. Launder, "On the Calculation of Turbulent Heat Transport Downstream from an Abrupt Pipe Expansion," Numerical Heat Transfer, vol. 3, no. 2, pp. 189-207, 1980.

[10] W. P. Jones, Be. Launder, "The prediction of laminarization with a two-equation model of turbulence," International Journal of Heat and Mass Transfer, vol. 15, no. 2, pp. 301-314, 1972.

[11] A. V. Gerasimov, "Development and validation of an analytical wall-function strategy for modelling forced, mixed and natural convection flows," Ph.D. thesis, University of Manchester : UMIST, 2004.

[12] K. Suga, T. J. Craft, H. Iacovides, "An analytical wall-function for turbulent flows and heat transfer over rough walls," International Journal of Heat and Fluid Flow, vol. 27, no. 5, pp. 852-866, 2006.

[13] D. C. Reda, J. D. Murphy, "Shock Wave/Turbulent Boundary-Layer Interactions in Rectangular Channels," AIAA Journal, vol. 11, no. 2, pp. 139-140, 1973.

[14] S. Pirozzoli, F. Grasso, "Direct numerical simulation of impinging shock wave/turbulent boundary layer interaction at M=2.25," Physics of Fluids, vol. 18, no. 6, pp. 65113, 2006.

[15] J. C. Chassaing, G. A. Gerolymos, I. Vallet, "Turbulence Structure Modification from Shock-Wave Micro Oscillations," in 37th AIAA Fluid Dynamics Conference and Exhibit, American Institute of Aeronautics and Astronautics. 